\title{
FINITE ELEMENT DISCRETIZATION OF THE KURAMOTO-SIVASHINSKY EQUATION
}

\author{
GEORGIOS D. AKRIVIS \\ Mathematics Department, University of Crete, 71409 Heraklion, Greece \\ and \\ Institute of Applied and Computational Mathematics \\ Research Center of Crete — FO.R.T.H., Heraklion, Greece
}

\begin{abstract}
We analyze semidiscrete and second-order in time fully discrete finite element methods for the Kuramoto-Sivashinsky equation.

1. Introduction. In this paper we study finite element approximations for the solution of the following periodic initial-value problem for the KuramotoSivashinsky (KS) equation: For $T, \nu>0$, we seek a real-valued function $u$ defined on $\mathbb{R} \times[0, T], 1$-periodic in the first variable and satisfying

$$
u_{t}+u u_{x}+u_{x x}+\nu u_{x x x x}=0 \quad \text { in } \mathbb{R} \times[0, T]
$$

$$
u(\cdot, 0)=u^{0} \quad \text { in } \mathbb{R},
$$

where $u^{0}$ is a given 1-periodic function. We assume that (1.1)-(1.2) has a unique, sufficiently smooth solution (cf. [8], [17]).

The KS equation was derived independently by Kuramoto and Sivashinsky in the late 70's and is related to turbulence phenomena in chemistry and combustion. It also arises in a variety of other physical problems such as plasma physics and two-phase flows in cylindrical geometries. For the mathematical theory and the physical significance of the KS equation as well as for related computational work we refer the reader to [7], [16], [3], [4], [17], [5], [6], [8], [9], [13], [14], [1] and the references therein; see also Temam [18] for an overview. In [1] the discretization of (1.1)-(1.2) by a Crank-Nicolson finite difference method and a linearization
\end{abstract}

1991 Mathematics Subject Classification: 65M60, 65M15.

The paper is in final form and no version of it will be published elsewhere. 
thereof by Newton's method is studied. In the present paper we analyze a semidiscrete method and a second-order in time fully discrete finite element method. The discretization in space is based on the standard Galerkin method; for the time discretization the Crank-Nicolson scheme is used.

For $m \in \mathbb{N}$ let $H_{\text {per }}^{m}$ be the periodic Sobolev space of order $m$, consisting of the 1-periodic elements of $H_{\mathrm{loc}}^{m}(\mathbb{R})$. We denote by $\|\cdot\|_{m}$ the norm over a period in $H_{\text {per }}^{m}$, by $\|\cdot\|$ the norm in $L^{2}(0,1)$, and by $(\cdot, \cdot)$ the inner product in $L^{2}(0,1)$. A variational form of (1.1) is

$$
\left(u_{t}, v\right)+\left(u u_{x}, v\right)-\left(u_{x}, v^{\prime}\right)+\nu\left(u_{x x}, v^{\prime \prime}\right)=0 \quad \forall v \in H_{\mathrm{per}}^{2} .
$$

Taking $v:=u(\cdot, t)$ in (1.3) we obtain by periodicity

$$
\frac{1}{2} \frac{d}{d t}\|u(\cdot, t)\|^{2}=\left\|u_{x}(\cdot, t)\right\|^{2}-\nu\left\|u_{x x}(\cdot, t)\right\|^{2} .
$$

Now, for $v \in H_{\mathrm{per}}^{2},\left\|v^{\prime}\right\|^{2}=-\left(v, v^{\prime \prime}\right)$, i.e.,

$$
\left\|v^{\prime}\right\|^{2} \leq\|v\|\left\|v^{\prime \prime}\right\|, \quad v \in H_{\mathrm{per}}^{2}
$$

Therefore,

$$
\left\|v^{\prime}\right\|^{2} \leq \nu\left\|v^{\prime \prime}\right\|^{2}+\frac{1}{4 \nu}\|v\|^{2}, \quad v \in H_{\mathrm{per}}^{2}
$$

and (1.4) leads to

i.e.,

$$
\frac{d}{d t}\|u(\cdot, t)\|^{2} \leq \frac{1}{2 \nu}\|u(\cdot, t)\|^{2}
$$

$$
\|u(\cdot, t)\| \leq\left\|u^{0}\right\| e^{t /(4 \nu)}, \quad 0 \leq t \leq T .
$$

Moreover, using the well-known Wirtinger inequality

$$
\left\|v^{\prime}\right\| \leq \frac{1}{2 \pi}\left\|v^{\prime \prime}\right\|, \quad v \in H_{\mathrm{per}}^{2},
$$

(cf. [12]), (1.4) yields

$$
\frac{1}{2} \frac{d}{d t}\|u(\cdot, t)\|^{2} \leq\left(\frac{1}{4 \pi^{2}}-\nu\right)\left\|u_{x x}(\cdot, t)\right\|^{2},
$$

and, consequently,

$$
\|u(\cdot, t)\| \leq\|u(\cdot, s)\|, \quad 0 \leq s \leq t \leq T, \quad \text { for } \nu \geq \frac{1}{4 \pi^{2}} .
$$

We shall discretize (1.1)-(1.2) in space by the standard Galerkin method. To this end, let $0=x_{0}<x_{1}<\ldots<x_{J}=1$ be a partition of $[0,1], h:=\max _{j}\left(x_{j+1}-\right.$ $\left.x_{j}\right)$, and $\underline{h}:=\min _{j}\left(x_{j+1}-x_{j}\right)$. Setting $x_{j J+s}:=x_{s}, j \in \mathbb{Z}, s=0, \ldots, J-1$, this partition is extended periodicaly to a partition of $\mathbb{R}$. For integer $r \geq 4$, let $S_{h}^{r}$ denote a space of continuously differentiable, 1-periodic splines of degree $r-1$ in which approximations to the solution $u(\cdot, t)$ of $(1.1)-(1.2)$ will be sought for 
$0 \leq t \leq T$. The following approximation property for the family $\left(S_{h}^{r}\right)_{0<h<1}$ is well known:

$$
\inf _{\chi \in S_{h}^{r}} \sum_{j=0}^{2} h^{j}\|v-\chi\|_{j} \leq c h^{s}\|v\|_{s}, \quad v \in H_{\mathrm{per}}^{s}, 2 \leq s \leq r,
$$

(cf., e.g., Schumaker [15], §8.1). Motivated by (1.3) we define the semidiscrete approximation $u_{h}(\cdot, t) \in S_{h}^{r}, 0 \leq t \leq T$, to $u$ by

$$
\left(u_{h t}, \chi\right)+\left(u_{h} u_{h x}, \chi\right)-\left(u_{h x}, \chi^{\prime}\right)+\nu\left(u_{h x x}, \chi^{\prime \prime}\right)=0 \quad \forall \chi \in S_{h}^{r},
$$

where $u_{h}(\cdot, 0):=u_{h}^{0} \in S_{h}^{r}$, and $u_{h}^{0}$ is such that

$$
\left\|u^{0}-u_{h}^{0}\right\| \leq c h^{r} .
$$

In Section 2 we show existence and uniqueness of the semidiscrete approximation, and derive the optimal-order error estimate

$$
\max _{0 \leq t \leq T}\left\|u(\cdot, t)-u_{h}(\cdot, t)\right\| \leq c h^{r} .
$$

In analogy to the exact solution, for the semidiscrete approximation the following inequalities hold:

$$
\left\|u_{h}(\cdot, t)\right\| \leq\left\|u_{h}^{0}\right\| e^{t /(4 \nu)}, \quad 0 \leq t \leq T,
$$

and

$$
\left\|u_{h}(\cdot, t)\right\| \leq\left\|u_{h}(\cdot, s)\right\|, \quad 0 \leq s \leq t \leq T, \quad \text { for } \nu \geq \frac{1}{4 \pi^{2}} .
$$

Section 3 is devoted to a second-order in time fully discrete finite element method for (1.1)-(1.2). Let $N \in \mathbb{N}, k:=T / N$, and $t^{n}:=n k, n=0, \ldots, N$. For $v(\cdot, t) \in L^{2}(0,1), 0 \leq t \leq T$, let

$$
v^{n}:=v\left(\cdot, t^{n}\right), \quad \partial v^{n}:=\frac{1}{k}\left(v^{n+1}-v^{n}\right), \quad \text { and } \quad v^{n+1 / 2}:=\frac{1}{2}\left(v^{n}+v^{n+1}\right) .
$$

The Crank-Nicolson approximations $U^{n} \in S_{h}^{r}$ to $u^{n}$ are then given by $U^{0}:=u_{h}^{0}$, and for $n=0, \ldots, N-1$

$$
\begin{aligned}
\left(\partial U^{n}, \chi\right)+\left(U^{n+1 / 2} U_{x}^{n+1 / 2}, \chi\right)-\left(U_{x}^{n+1 / 2}, \chi^{\prime}\right)+\nu\left(U_{x x}^{n+1 / 2}, \chi^{\prime \prime}\right) & =0 \\
& \forall \chi \in S_{h}^{r} .
\end{aligned}
$$

The following discrete analogs to (1.7) and (1.8), respectively, can be easily proved:

$$
\left\|U^{n}\right\| \leq\left\|U^{0}\right\| e^{\alpha /(4 \nu) t^{n}}, \quad \alpha>1, k \leq 8 \nu \frac{\alpha-1}{\alpha}, n=1, \ldots, N,
$$

and

$$
\left\|U^{n+1}\right\| \leq\left\|U^{n}\right\|, \quad n=0, \ldots, N-1, \quad \text { for } \nu \geq \frac{1}{4 \pi^{2}} .
$$


Further, we show existence of the Crank-Nicolson approximations for $k<8 \nu$, and derive the optimal-order error estimate

$$
\max _{0 \leq n \leq N}\left\|u^{n}-U^{n}\right\| \leq c\left(k^{2}+h^{r}\right) .
$$

We also prove uniqueness of the fully discrete approximations under a mild mesh condition.

It is well known and easily seen that $u(\cdot, t)$ is odd for $0 \leq t \leq T$ if the initial value $u^{0}$ is an odd function. This property carries over to the semidiscrete and the fully discrete approximations provided $\chi \in S_{h}^{r}$ implies $\chi(-\cdot) \in S_{h}^{r}$.

2. Semidiscretization. In this section we briefly study the semidiscrete approximation $u_{h}$. The inequality (1.14) can be proved in the same way as (1.7). Now, it is evident from (1.14) and the fact that $S_{h}^{r}$ is finite-dimensional that an estimate of the form

$$
\max _{0 \leq t \leq T}\left\|u_{h}(\cdot, t)\right\|_{L^{\infty}} \leq c(h)
$$

is valid. Combining this with the fact that the "right-hand side" of the system of O.D.E.'s (1.11) is locally Lipschitz continuous we deduce existence and uniqueness of the semidiscrete approximation $u_{h}$.

In the error estimation that follows we will compare the semidiscrete approximation with the elliptic projection of the exact solution. This projection $P_{E}: H_{\text {per }}^{2} \rightarrow S_{h}^{r}$ is defined by

$$
\nu\left(v^{\prime \prime}-\left(P_{E} v\right)^{\prime \prime}, \chi^{\prime \prime}\right)-\left(v^{\prime}-\left(P_{E} v\right)^{\prime}, \chi^{\prime}\right)+\lambda\left(v-\left(P_{E} v\right), \chi\right)=0 \quad \forall \chi \in S_{h}^{r},
$$

where $\lambda>1 /(2 \nu)$. For the elliptic projection we have the following estimate:

$$
\sum_{j=0}^{2} h^{j}\left\|v-P_{E} v\right\|_{j} \leq c h^{s}\|v\|_{s}, \quad v \in H_{\mathrm{per}}^{s}, 2 \leq s \leq r
$$

(cf. [11]). This estimate can be proved in the usual manner. First, using the fact that the bilinear form $a$,

$$
a(v, w):=\nu\left(v^{\prime \prime}, w^{\prime \prime}\right)-\left(v^{\prime}, w^{\prime}\right)+\lambda(v, w),
$$

is continuous and coercive in $H_{\text {per }}^{2}$ (cf. (1.5)), the Lax-Milgram lemma yields, in view of the approximation property (1.10),

$$
\left\|v-P_{E} v\right\|_{2} \leq c h^{s-2}\|v\|_{s}, \quad v \in H_{\mathrm{per}}^{s}, 2 \leq s \leq r .
$$

Next, to estimate $\left\|v-P_{E} v\right\|$ consider the auxiliary problem

$$
a(\psi, w)=\left(v-P_{E} v, w\right) \quad \forall w \in H_{\mathrm{per}}^{2} .
$$

Then, for $\chi \in S_{h}^{r}$ we have

$$
\left\|v-P_{E} v\right\|^{2}=a\left(\psi-\chi, v-P_{E} v\right) \leq c\|\psi-\chi\|_{2}\left\|v-P_{E} v\right\|_{2} .
$$


Therefore, the well-known regularity estimate $\|\psi\|_{4} \leq c\left\|v-P_{E} v\right\|$, easily established in our one-dimensional case, and (1.10), (2.3) yield

$$
\left\|v-P_{E} v\right\| \leq c h^{s}\|v\|_{s}, \quad v \in H_{\mathrm{per}}^{s}, 2 \leq s \leq r .
$$

The estimate (2.2) now follows from (2.3), (2.4) and (1.5).

THEOREM 2.1. Let the solution $u$ of (1.1)-(1.2) be sufficiently smooth, and let (1.12) hold. Then

$$
\max _{0 \leq t \leq T}\left\|u(\cdot, t)-u_{h}(\cdot, t)\right\| \leq c h^{r} .
$$

Proof. Let $W(\cdot, t):=P_{E} u(\cdot, t), \varrho(\cdot, t):=u(\cdot, t)-W(\cdot, t)$, and $\vartheta(\cdot, t):=$ $W(\cdot, t)-u_{h}(\cdot, t)$. Then $u-u_{h}=\varrho+\vartheta$ and by $(2.2)$

$$
\max _{0 \leq t \leq T}\|\varrho(\cdot, t)\| \leq c h^{r} \text {. }
$$

Thus, it remains to estimate $\|\vartheta(\cdot, t)\|$. Using (1.11), (2.1) and (1.3) we have, for $\chi \in S_{h}^{r}$,

$$
\begin{aligned}
\left(\vartheta_{t}, \chi\right)+a(\vartheta, \chi) & =\left(W_{t}, \chi\right)+a(W, \chi)-\left(u_{h t}, \chi\right)-a\left(u_{h}, \chi\right) \\
& =\left(W_{t}, \chi\right)+a(u, \chi)+\left(u_{h} u_{h x}, \chi\right)-\lambda\left(u_{h}, \chi\right) \\
& =\left(\lambda \varrho-\varrho_{t}, \chi\right)-\left(u u_{x}-u_{h} u_{h x}, \chi\right)+\lambda(\vartheta, \chi)
\end{aligned}
$$

i.e.,

$$
\begin{aligned}
&\left(\vartheta_{t}, \chi\right)+\nu\left(\vartheta_{x x}, \chi^{\prime \prime}\right)-\left(\vartheta_{x}, \chi^{\prime}\right) \\
&=\left(\lambda \varrho-\varrho_{t}+\varrho \varrho_{x}+\vartheta \vartheta_{x}, \chi\right)+\left(u \varrho+W \vartheta, \chi^{\prime}\right) \quad \forall \chi \in S_{h}^{r}
\end{aligned}
$$

A straightforward consequence of the commutativity of $P_{E}$ with time differentiation is

$$
\max _{0 \leq t \leq T}\left\|\varrho_{t}(\cdot, t)\right\| \leq c h^{r} .
$$

Further, (2.2) yields in our one-dimensional case

$$
\max _{0 \leq t \leq T}\|W(\cdot, t)\|_{L^{\infty}} \leq c .
$$

Taking $\chi:=\vartheta(\cdot, t)$ in $(2.7)$ and using $(2.6),(2.8)$ and (2.9) we obtain by periodicity

$$
\frac{1}{2} \frac{d}{d t}\|\vartheta(\cdot, t)\|^{2}+\nu\left\|\vartheta_{x x}\right\|^{2}-\left\|\vartheta_{x}\right\|^{2} \leq c h^{2 r}+c\|\vartheta\|^{2}+\left\|\vartheta_{x}\right\|^{2} .
$$

Therefore, using (1.5) we obtain

$$
\frac{1}{2} \frac{d}{d t}\|\vartheta(\cdot, t)\|^{2} \leq c h^{2 r}+c\|\vartheta\|^{2}
$$

and Gronwall's lemma yields, in view of (1.12),

$$
\max _{0 \leq t \leq T}\|\vartheta(\cdot, t)\| \leq c h^{r}
$$

which concludes the proof. 
3. Crank-Nicolson discretization. In this section we show existence of the Crank-Nicolson approximations $U^{1}, \ldots, U^{N}$ for $k<8 \nu$, derive the optimal-order error estimate (1.19), and under a mild mesh condition prove uniqueness of the Crank-Nicolson approximations. We also briefly discuss the case of an odd initial value.

Taking $\chi:=U^{n+1 / 2}$ in $(1.16)$ we obtain by periodicity

$$
\left\|U^{n+1}\right\|^{2}-\left\|U^{n}\right\|^{2}=2 k\left\{\left\|U_{x}^{n+1 / 2}\right\|^{2}-\nu\left\|U_{x x}^{n+1 / 2}\right\|^{2}\right\},
$$

and (1.18) follows using (1.8). Further, using (1.6) we obtain from (3.1),

$$
\left\|U^{n+1}\right\|^{2}-\left\|U^{n}\right\|^{2} \leq \frac{k}{2 \nu}\left\|U^{n+1 / 2}\right\|^{2},
$$

i.e.,

$$
\left(1-\frac{k}{8 \nu}\right)\left\|U^{n+1}\right\| \leq\left(1+\frac{k}{8 \nu}\right)\left\|U^{n}\right\|, \quad n=0, \ldots, N-1 .
$$

For $\alpha>1$ obviously

$$
\frac{8 \nu+k}{8 \nu-k} \leq 1+\frac{\alpha}{4 \nu} k \quad \text { for } k \leq 8 \nu \frac{\alpha-1}{\alpha},
$$

and (1.17) follows easily from (3.2).

Existence. We shall use the following well-known variant of the Brouwer fixedpoint theorem (see, e.g., Browder [2]).

Lemma 3.1. Let $\left(H,(\cdot, \cdot)_{H}\right)$ be a finite-dimensional inner product space and denote by $\|\cdot\|_{H}$ the induced norm. Suppose that $g: H \rightarrow H$ is continuous and there exists an $\alpha>0$ such that $(g(x), x)_{H}>0$ for all $x \in H$ with $\|x\|_{H}=\alpha$. Then there exists $x^{*} \in H$ such that $g\left(x^{*}\right)=0$ and $\left\|x^{*}\right\| \leq \alpha$.

The proof of existence of $U^{0}, \ldots, U^{N}$ for $k<8 \nu$ is by induction. Assume that $U^{0}, \ldots, U^{n}, n<N$, exist and let $g: S_{h}^{r} \rightarrow S_{h}^{r}$ be defined by

$$
(g(V), \chi)=2\left(V-U^{n}, \chi\right)+k\left(V V^{\prime}, \chi\right)-k\left(V^{\prime}, \chi^{\prime}\right)+\nu k\left(V^{\prime \prime}, \chi^{\prime \prime}\right) \quad \forall V, \chi \in S_{h}^{r} .
$$

This mapping is obviously continuous. Furthermore, by periodicity we have

$$
(g(V), V)=2\left(V-U^{n}, V\right)-k\left\{\left\|V^{\prime}\right\|^{2}-\nu\left\|V^{\prime \prime}\right\|^{2}\right\},
$$

and via (1.6),

$$
(g(V), V) \geq 2\|V\|\left\{\left(1-\frac{k}{8 \nu}\right)\|V\|-\left\|U^{n}\right\|\right\} \quad \forall V \in S_{h}^{r} .
$$

Therefore, assuming $k<8 \nu$, for $\|V\|=\frac{8 \nu}{8 \nu-k}\left\|U^{n}\right\|+1$ obviously $(g(V), V)>0$ and the existence of a $V^{*} \in S_{h}^{r}$ such that $g\left(V^{*}\right)=0$ follows from Lemma 3.1. Then $U^{n+1}:=2 V^{*}-U^{n}$ satisfies (1.16).

Convergence. The main result in this paper is given in the following theorem. 
THEOREM 3.1. Let the solution $u$ of (1.1)-(1.2) be sufficiently smooth, $U^{0}, \ldots$ $\ldots, U^{N}$ satisfy (1.16), and (1.12) hold. Then, for $k$ sufficiently small,

$$
\max _{0 \leq n \leq N}\left\|u^{n}-U^{n}\right\| \leq c(u)\left(k^{2}+h^{r}\right) .
$$

Proof. Let $W^{n}:=W\left(\cdot, t^{n}\right), \varrho^{n}:=u^{n}-W^{n}$, and $\zeta^{n}:=W^{n}-U^{n}$. Then $u^{n}-U^{n}=\varrho^{n}+\zeta^{n}$ and by $(2.6)$,

$$
\max _{0 \leq n \leq N}\left\|\varrho^{n}\right\| \leq c h^{r} .
$$

Thus it remains to estimate $\left\|\zeta^{n}\right\|$. Using (1.16), (2.1) and (1.3) we have, for $\chi \in S_{h}^{r}$,

i.e.,

$$
\begin{aligned}
\left(\partial \zeta^{n}, \chi\right)+ & a\left(\zeta^{n+1 / 2}, \chi\right)=\left(\partial W^{n}, \chi\right)+a\left(W^{n+1 / 2}, \chi\right)-\left(\partial U^{n}, \chi\right)-a\left(U^{n+1 / 2}, \chi\right) \\
= & \left(\partial W^{n}, \chi\right)+a\left(u^{n+1 / 2}, \chi\right)+\left(U^{n+1 / 2} U_{x}^{n+1 / 2}, \chi\right)-\lambda\left(U^{n+1 / 2}, \chi\right) \\
= & \left(\partial W^{n}-u_{t}^{n+1 / 2}-\frac{1}{2}\left(u^{n} u_{x}^{n}+u^{n+1} u_{x}^{n+1}\right)\right. \\
& \left.+\lambda \varrho^{n+1 / 2}+\lambda \zeta^{n+1 / 2}+U^{n+1 / 2} U_{x}^{n+1 / 2}, \chi\right),
\end{aligned}
$$

$$
\begin{aligned}
\left(\partial \zeta^{n}, \chi\right)+\nu\left(\zeta_{x x}^{n+1 / 2}, \chi^{\prime \prime}\right)- & \left(\zeta_{x}^{n+1 / 2}, \chi^{\prime}\right) \\
= & \left(\omega^{n}+\varrho^{n+1 / 2} \varrho_{x}^{n+1 / 2}+\zeta^{n+1 / 2} \zeta_{x}^{n+1 / 2}, \chi\right) \\
& +\left(u^{n+1 / 2} \varrho^{n+1 / 2}+W^{n+1 / 2} \zeta^{n+1 / 2}, \chi^{\prime}\right),
\end{aligned}
$$

where $\omega^{n}=\omega_{1}^{n}+\omega_{2}^{n}+\omega_{3}^{n}+\lambda \varrho^{n+1 / 2}$, and

$$
\begin{aligned}
& \omega_{1}^{n}:=\partial W^{n}-\partial u^{n}, \\
& \omega_{2}^{n}:=\partial u^{n}-u_{t}^{n+1 / 2}, \\
& \omega_{3}^{n}:=u^{n+1 / 2} u_{x}^{n+1 / 2}-\frac{1}{2}\left(u^{n} u_{x}^{n}+u^{n+1} u_{x}^{n+1}\right) .
\end{aligned}
$$

It is easily seen that

$$
\max _{0 \leq n \leq N}\left\|\omega^{n}\right\| \leq c\left(k^{2}+h^{r}\right) .
$$

Taking $\chi:=\zeta^{n+1 / 2}$ in (3.5) and using (3.4), (3.6) and (2.9) we obtain by periodicity

$$
\begin{aligned}
\frac{1}{2 k}\left(\left\|\zeta^{n+1}\right\|^{2}-\left\|\zeta^{n}\right\|^{2}\right)+\nu\left\|\zeta_{x x}^{n+1 / 2}\right\|^{2} & -\left\|\zeta_{x}^{n+1 / 2}\right\|^{2} \\
& \leq c\left(k^{2}+h^{r}\right)^{2}+c\left\|\zeta^{n+1 / 2}\right\|^{2}+\left\|\zeta_{x}^{n+1 / 2}\right\|^{2} .
\end{aligned}
$$

Therefore by (1.5) we see that

$$
\left\|\zeta^{n+1}\right\|^{2}-\left\|\zeta^{n}\right\|^{2} \leq c k\left\{\left(k^{2}+h^{r}\right)^{2}+\left\|\zeta^{n+1}\right\|^{2}+\left\|\zeta^{n}\right\|^{2}\right\}
$$

and the discrete Gronwall lemma yields in view of (1.12) for $k$ sufficiently small

$$
\max _{0 \leq n \leq N}\left\|\zeta^{n}\right\| \leq c\left(k^{2}+h^{r}\right),
$$

which concludes the proof. 
Uniqueness. In addition to our assumptions on $S_{h}^{r}$ we suppose here for the corresponding partition that for a positive constant $\mu$,

$$
\underline{h} \geq c h^{2 \mu} \text {. }
$$

It is well known that this inequality implies

$$
\|\chi\|_{L^{\infty}} \leq c h^{-\mu}\|\chi\| \quad \forall \chi \in S_{h}^{r},
$$

(cf. Nitsche [10]). Let now $V^{0}=U^{0}$ and $V^{0}, \ldots, V^{N} \in S_{h}^{r}$ satisfy

$$
\begin{aligned}
\left(\partial V^{n}, \chi\right)+\left(V^{n+1 / 2} V_{x}^{n+1 / 2}, \chi\right)-\left(V_{x}^{n+1 / 2}, \chi^{\prime}\right)+\nu\left(V_{x x}^{n+1 / 2}, \chi^{\prime \prime}\right) & =0 \\
\forall \chi & \in S_{h}^{r},
\end{aligned}
$$

for $n=0, \ldots, N-1$. Letting $E^{n}:=U^{n}-V^{n}, n=0, \ldots, N$, from (1.16), (3.10) we obtain

$$
\begin{aligned}
\left(\partial E^{n}, \chi\right)+\nu\left(E_{x x}^{n+1 / 2}, \chi^{\prime \prime}\right)-\left(E_{x}^{n+1 / 2}, \chi^{\prime}\right) & \\
& =\left(E^{n+1 / 2} E_{x}^{n+1 / 2}, \chi\right)+\left(U^{n+1 / 2} E^{n+1 / 2}, \chi^{\prime}\right) \quad \forall \chi \in S_{h}^{r} .
\end{aligned}
$$

Taking $\chi:=E^{n+1 / 2}$ we obtain by periodicity

$$
\begin{aligned}
\frac{1}{2 k}\left(\left\|E^{n+1}\right\|^{2}-\|\right. & \left.E^{n} \|^{2}\right)+\nu\left\|E_{x x}^{n+1 / 2}\right\|^{2}-\left\|E_{x}^{n+1 / 2}\right\|^{2} \\
& =\left(U^{n+1 / 2} E^{n+1 / 2}, E_{x}^{n+1 / 2}\right) \\
& \leq \frac{1}{2}\left(\left\|W^{n+1 / 2}\right\|_{L^{\infty}}^{2}+\left\|\zeta^{n+1 / 2}\right\|_{L^{\infty}}^{2}\right)\left\|E^{n+1 / 2}\right\|^{2}+\left\|E_{x}^{n+1 / 2}\right\|^{2} \\
& \leq\left(c+c h^{-2 \mu}\left(k^{4}+h^{2 r}\right)\right)\left\|E^{n+1 / 2}\right\|^{2}+\left\|E_{x}^{n+1 / 2}\right\|^{2}
\end{aligned}
$$

where (2.9), (3.9) and (3.7) have been used. Then (1.5) yields

(3.11) $\quad\left\|E^{n+1}\right\|^{2}-\left\|E^{n}\right\|^{2} \leq C k\left(1+k^{4} h^{-2 \mu}+h^{2(r-\mu)}\right)\left(\left\|E^{n+1}\right\|^{2}+\left\|E^{n}\right\|^{2}\right)$.

For $k^{5} h^{-2 \mu}$ and $k h^{2(r-\mu)}$ sufficiently small, assuming $E^{n}=0$, (3.11) implies $E^{n+1}=0$. Summarizing, for sufficiently smooth $u$ and $k^{5} h^{-2 \mu}, k h^{2(r-\mu)}$ sufficiently small, assuming (3.9) we deduce uniqueness of the Crank-Nicolson approximations.

Odd initial value. We assume here that the initial value $u^{0}$ is an odd function. Then $v(x, t):=-u(-x, t)$ is a solution of $(1.1)-(1.2)$. Thus $v=u$, i.e., $u(\cdot, t)$ is odd for $0 \leq t \leq T$.

Assume now that if $x_{i}$ is a knot of our spline space then $-x_{i}$ is a knot as well, and moreover that the same differentiability conditions are posed at $x_{i}$ and $-x_{i}$, $i \in \mathbb{Z}$. As a consequence, $\chi \in S_{h}^{r}$ implies $\chi(-\cdot) \in S_{h}^{r}$. Let $u_{h}^{0}$ be an odd function as is natural for odd $u^{0}$. Then the semidiscrete approximation $u_{h}(\cdot, t)$ is odd for $0 \leq t \leq T$, and moreover under our assumptions implying uniqueness of the Crank-Nicolson approximations $U^{n}$, they are odd, since $V^{n}:=-U^{n}(-\cdot)$ are also Crank-Nicolson approximations. This fact is of significant practical importance, since in (1.16) we only have to take the odd $\chi$ 's thus reducing the number of equations to about $50 \%$. 


\section{References}

[1] G. D. Akrivis, Finite difference discretization of the Kuramoto-Sivashinsky equation, Numer. Math. 63 (1992), 1-11.

[2] F. E. Browder, Existence and uniqueness theorems for solutions of nonlinear boundary value problems, in: Applications of Nonlinear Partial Differential Equations, R. Finn (ed.), Proc. Sympos. Appl. Math. 17, Amer. Math. Soc., Providence 1965, 24-49.

[3] P. Constantin, C. Foiaş, B. Nicolaenko and R. Temam, Integral Manifolds and Inertial Manifolds for Dissipative Partial Differential Equations, Springer, New York 1989.

[4] J. M. Hyman and B. Nicolaenko, The Kuramoto-Sivashinsky equation: A bridge between PDE's and dynamical systems, Phys. D 18 (1986), 113-126.

[5] J. M. Jolly, I. G. Kevrekidis and E. S. Titi, Approximate inertial manifolds for the Kuramoto-Sivashinsky equation; analysis and computations, ibid. 44 (1990), 38-60.

[6] I. G. Kevrekidis, B. Nicolaenko and J. C. Scovel, Back in the saddle again; a computer assisted study of the Kuramoto-Sivashinsky equation, SIAM J. Appl. Math. 50 (1990), 760-790

[7] Y. Kuramoto, Diffusion induced chaos in reaction systems, Progr. Theoret. Phys. Suppl. 64 (1978), 346-367.

[8] B. Nicolaenko and B. Scheurer, Remarks on the Kuramoto-Sivashinsky equation, Phys. D 12 (1984), 391-395.

[9] B. Nicolaenko, B. Scheurer and R. Temam, Some global dynamical properties of the Kuramoto-Sivashinsky equation: Nonlinear stability and attractors, ibid. 16 (1985), $155-183$.

[10] J. Nitsche, Umkehrsätze für Spline-Approximationen, Compositio Math. 21 (1969), 400416.

[11] _-, Verfahren von Ritz und Spline-Interpolation bei Sturm-Liouville-Randwertproblemen, Numer. Math. 13 (1969), 260-265.

[12] R. Osserman, The isoperimetric inequality, Bull. Amer. Math. Soc. 84 (1978), 11821238.

[13] D. T. Papageorgiou, C. Maldarelli and D. S. Rumschitzki, Nonlinear interfacial stability of core-annular film flows, Phys. Fluids A2 (1990), 340-352.

[14] D. T. Papageorgiou and Y. S. Smyrlis, The route to chaos for the Kuramoto-Sivashinsky equation, Theoret. Comput. Fluid Dynamics 3 (1991), 15-42.

[15] L. L. Schumaker, Spline Functions: Basic Theory, Wiley, New York 1981.

[16] G. I. Sivashinsky, On flame propagation under conditions of stoichiometry, SIAM J. Appl. Math. 39 (1980), 67-82.

[17] E. Tadmor, The well-posedness of the Kuramoto-Sivashinsky equation, SIAM J. Math. Anal. 17 (1986), 884-893.

[18] R. Temam, Infinite-Dimensional Dynamical Systems in Mechanics and Physics, Springer, New York 1988. 